\title{
Câncer de reto durante gestação: relato de caso e revisão da literatura
}

\section{Rectal cancer during pregnancy: case report and literature review}

\author{
ANTÔNIO HILÁRIO ALVES FREITAS ${ }^{1}$, HUMBERTO MADEIRA² ${ }^{2}$ LEONARDO MACIEL DA FONSECA, \\ EDUARDO VITOR DE CASTRO ${ }^{4}$
}

\begin{abstract}
${ }^{1}$ Coloproctologista do Hospital Alberto Cavalcanti; Membro titular da SBCP - Belo Horizonte (MG), Brasil. ${ }^{2}$ Obstetra da Maternidade Odete Valadares - Belo Horizonte $(M G)$, Brasil. ${ }^{3}$ Coloproctologista e Mestrando na Universidade Federal de Minas Gerais (UFMG) - Belo Horizonte (MG), Brasil. ${ }^{4}$ Médico residente do Hospital Alberto Cavalcanti - Belo Horizonte (MG), Brasil.
\end{abstract}

FREITAS AHA, MADEIRA H, FONSECA LMD, CASTRO EVD. Câncer de reto durante gestação: relato de caso e revisão da literatura. Rev bras Coloproct, 2011;31(1): 81-84.

RESUMO: $\mathrm{O}$ carcinoma colorretal associado à gestação é um acontecimento raro, relacionado a um prognóstico ruim para as mulheres. $O$ caso descrito é de uma paciente, do sexo feminino, de 31 anos, com adenocarcinoma de reto diagnosticado na $24^{\mathrm{a}}$ semana gestacional. É apresentada uma discussão sobre conduta e atualização de abordagem terapêutica.

Palavras-chave: câncer de cólon; câncer de reto, gravidez.

\section{INTRODUÇÃO}

O carcinoma colorretal (CCR) é o terceiro em incidência e mortalidade entre as mulheres em idade reprodutiva. A incidência de câncer em gestantes que foi descrita na literatura varia de 0,07 a $0,1 \%$. O CCR durante o período gestacional é uma entidade rara, com incidência de $0,002 \%{ }^{1-12}$.

É uma condição que envolve aspectos éticos, religiosos e morais, podendo repercutir tanto na abordagem quanto no tratamento.

Sabe-se que apresenta prognóstico ruim para mãe, desde os relatos de Cruveilhier (1842) e Evers (1928), os quais descreveram os primeiros casos de tumores colorretais na gravidez ${ }^{2,4}$. De acordo com a literatura, nenhuma paciente com CCR na gestação alcançou sobrevida de cinco anos ${ }^{13}$. Contudo, as pers- pectivas para o feto são boas, considerando-se que não há relato de metástases para o mesmo, acrescentandose ainda as melhorias nos cuidados neonatais.

\section{RELATO DE CASO}

Paciente de 31 anos, sexo feminino, na $24^{\mathrm{a}}$ semana de gestação, apresentou-se na Unidade de Emergência do Hospital Alberto Cavalcanti (HAC) com queixa de afilamento do calibre das fezes, associada à hematoquezia iniciada há 30 dias. Ela não apresentava perda de peso ou sintomas de obstrução intestinal. Ao exame proctológico, notou-se lesão tumoral circunferencial, mais proeminente em parede anterior do reto, exofítica, com abscesso drenando secreção purulenta na região anoperineal. Foram realizadas biópsias que mostraram adenocarcinoma moderadamente diferen-

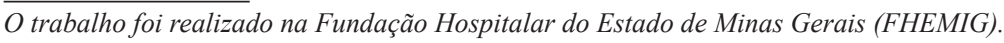

Recebido em: 02/03/2009

Aprovado em: 14/04/2011 
ciado invasivo, com presença de desmoplasia do estroma (Figura 1).

A avaliação obstétrica confirmou tratar-se de uma gestação de 24 semanas, com feto em boas condições. Havia também invasão tumoral da parede vaginal posterior, com formação de fístula retovaginal.

Durante avaliação propedêutica, constatou-se anemia, antígeno carcinoembrionário (CEA) de 2,5 $\mathrm{ng} / \mathrm{mL}$, e volumosa massa anorretal na ressonância magnética mostrando invasão de todas as camadas do reto, em situação anterolateral direita, sem plano de clivagem com parede vaginal posterior. Houve comprometimento do complexo esfincteriano e presença de linfadenomegalia perirretal bilateral. Útero e bexiga sem sinais de invasão tumoral (Figura 2).

A gestação da paciente foi conduzida conjuntamente com a equipe de obstetrícia da Maternidade Odete Valadares (MOV). Atingida a maturidade pulmonar fetal, foi realizada cesariana na $27^{\mathrm{a}}$ semana, sem intercorrências para a mãe e o recém-nascido, sendo este conduzido ao berçário de alto risco.

Uma semana após o parto, a paciente foi reconduzida ao HAC e submetida à laparotomia. $\mathrm{O}$ inventário da cavidade abdominal não evidenciou doença metastática macroscópica. Foram realizadas histerectomia com salpingo-ooforectomia bilateral, associada à ressecção em bloco do retossigmoide, paredes lateral e posterior da vagina, e circunferência em torno do ânus com $10 \mathrm{~cm}$ de diâmetro. $\mathrm{O}$ peritônio abdominal foi suturado, e a abertura do períneo deixada para fechamento por segunda intenção. Houve colostomia definitiva em quadrante inferior esquerdo do abdome.

O anatomopatológico da peça cirúrgica mostrou adenocarcinoma retal invasivo, moderadamente diferenciado, vegetante ulcerado, comprometendo toda circunferência intestinal, estendendo-se do canal anal até $7 \mathrm{~cm}$ no reto, e três linfonodos positivos para metástases. $\mathrm{O}$ estadiamento patológico foi $\mathrm{T}_{4} \mathrm{~N}_{1} \mathrm{M}_{0}$ (Dukes C).

A criança teve boa evolução, inicialmente, recebeu cuidados no berçário de alto risco, depois ela foi encaminhada para o quarto e, finalmente, hígida, recebeu alta hospitalar, tendo acompanhado a paciente nas consultas ambulatoriais.

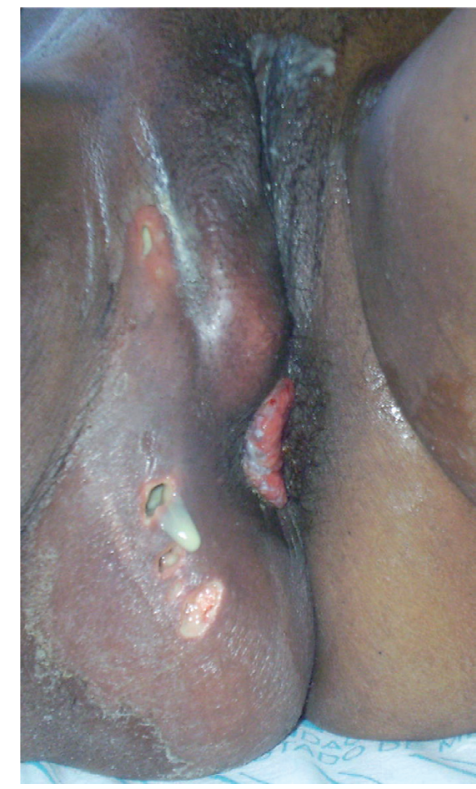

Figura 1. Lesão tumoral do reto, exofítica, com abscesso.

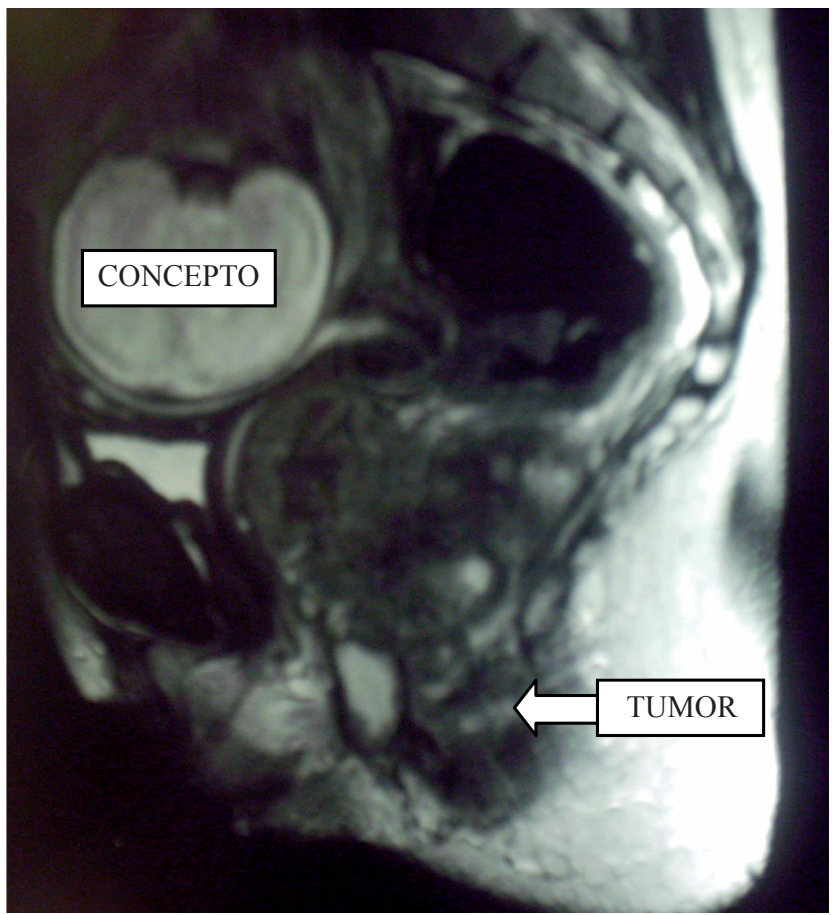

Figura 2. Ressonância nuclear magnética mostrando gravidez e tumor de reto.

A mãe evoluiu sem intercorrências, recebendo alta após nove dias da cirurgia. Foram realizadas radio e quimioterapia adjuvantes. Alcançou sobrevida de 26 meses, e teve como causa de óbito doença metastática em fígado e pulmões. 


\section{DISCUSSÃO}

O CCR durante a gravidez é uma doença rara. Até o momento, foram descritos na literatura cerca de 250 casos, e o prognóstico para a mãe em todos os relatos é bastante reservado ${ }^{1,2,4,5}$. O aumento na incidência poderia ser atribuído à idade mais avançada em que as mulheres estão engravidando ${ }^{1,2,8,9}$. Geralmente, o diagnóstico é tardio e os sintomas são confundidos com os de uma gestação normal. A abordagem do paciente torna-se um desafio para o médico assistente, que objetiva realizar um tratamento curativo, resguardando o bem-estar fetal e permeando questões éticas, morais e religiosas.

A influência da gestação na carcinogênese é desconhecida. Não é conclusivo que a elevação dos níveis de estrógeno, progesterona e prolactina durante este período possam estimular o crescimento tumoral.

Diferentemente da população em geral, durante a gravidez, o CCR tem predominância pelo reto, sendo que, em $80 \%$ dos casos, localiza-se abaixo da reflexão peritoneal. Nos tumores acima da reflexão peritoneal, a distribuição é semelhante à da população. Esta predominância de acometimento retal não é totalmente entendida, sendo aventada a hipótese de maior realização de exames pélvicos durante a gestação ${ }^{1,2,4,5,9,10}$.

Para diagnóstico precoce, é necessário alto índice de suspeição. Como há predominância de tumores localizados no reto, o toque retal, a anuscopia e a retossigmoidoscopia são primordiais, seguidos de colonoscopia, quando a fase gestacional permite, para pesquisa de tumores sincrônicos. Em relação aos exames de imagens, a ultrassonografia pode ser utilizada por ser inócua, mas a ressonância nuclear magnética da pelve e abdome fornece informações mais precisas, sem causar malefícios ao feto. O enema opaco e a tomografia computadorizada são contraindicados, exceto nas fases mais tardias da gestação. A dosagem do CEA pode estar elevada na gravidez normal, não sendo usada para diagnóstico, mas é válida para seguimento pós-operatório.

O tratamento é complexo e envolve aspectos como idade gestacional, estadiamento tumoral, preservação da fertilidade e, ainda, questões de ordem pessoal, moral e ética. O tratamento curativo é a ressecção cirúrgica com margens livres, associada à linfadenectomia oncológica. Há autores que defendem o tratamento semelhante ao de uma mulher não-grávida. Contudo, a conduta indicada pela maioria dos autores é baseada na idade gestacional em que foi feito o diagnóstico.

Até a primeira metade da gestação, o tratamento deve ser realizado como o de uma paciente não-grávida. $\mathrm{O}$ atraso do tratamento até a viabilidade fetal pode resultar no crescimento do tumor e no surgimento de metástases. O tipo de ressecção varia de acordo com os achados cirúrgicos. O útero é preservado caso não impeça o acesso à pelve ou não apresente invasão tumoral macroscópica. Os relatos de preservação e desenvolvimento fetal após ressecção tumoral são raros. A necessidade de salpingo-ooforectomia é associada a altos índices de perda fetal. Também não há conclusões claras sob o efeito da cirurgia e da anestesia em relação à viabilidade e ao desenvolvimento fetal ${ }^{4}$.

$\mathrm{Na}$ segunda metade, aguarda-se a viabilidade fetal e, após o parto, realiza-se a cirurgia. A via de parto, se vaginal ou cesariana, segue indicações obstétricas, sendo as mesmas de uma paciente hígida. O parto vaginal é preferido. Contudo, tumores retais baixos, que obstruem o canal do parto ou que estão localizados na parede anterior do reto e que possam causar trauma ao feto ou mesmo ser atingido durante a episiotomia, contraindicam esta via. Caso haja necessidade de realização de cesariana, a ressecção tumoral pode ser realizada no mesmo tempo cirúrgico, ou a cirurgia pode ser adiada por alguns dias para que ocorram regressão uterina e diminuição da congestão vascular pélvica, minimizando o risco de sangramento.

A ooforectomia é recomendada pela maioria dos autores pela alta incidência de metástases, principalmente quando o reto é acometido. Na população em geral, o índice de metástases ovarianas é de 3 a $8 \%$, enquanto em grávidas esse número aumenta para aproximadamente $24 \%^{1,2,46}$. Uma conduta defendida é a realização de biópsias de congelação durante $\mathrm{o}$ ato operatório, quando a paciente deseja preservar a fertilidade, e caso positiva realiza-se salpingo-ooforectomia associada à histerectomia. $\mathrm{O}$ prognóstico de pacientes com metástases ovarianas é reservado, com uma sobrevida de apenas entre 3 a 12 meses $^{4,6}$.

As principais drogas utilizadas na quimioterapia adjuvante são o 5-fluouracil e levamisol. A associação destas duas drogas após cirurgia curativa tem trazido bons resultados para pacientes com tumores Dukes C, 
reduzindo a recorrência em cerca de $41 \%$ e a mortalidade em $33 \%^{1,4}$. Não são conhecidos os efeitos do uso destas drogas no início da gestação, sendo consideradas seguras a partir do segundo trimestre, sem aumentar a chance de perda ou de desenvolvimento de anormalidades no feto, mas há risco de prematuridade e retardo de desenvolvimento.

Em relação à radioterapia, é sabidamente benéfica tanto no pré como no pós-operatório dos tumores de reto. Contudo, é contraindicada durante a gestação, e seus efeitos para o feto são imprevisíveis. A paciente também deve ser alertada que a irradiação da pelve leva a uma castração funcional. Como terapia adjuvante, em associação com quimioterapia, é indicada para tumores estágio II e III, da mesma forma que em pacientes não-grávidas, sendo que não há protocolos definidos para gestantes com tumor de reto.

\section{CONCLUSÃO}

O prognóstico dos CCR em gestantes varia de acordo com estádio em que o tumor é diagnosticado. Uma evolução ruim pode ser atribuída ao atraso diagnóstico devido aos sintomas gastrintestinais serem confundidos com os de uma gestação normal ou com doenças benignas. Não há evidências de que a gestação altere o curso da doença, mas alguns autores defendem que as alterações hormonais e imunes que ocorrem durante a gestação possam promover rápido crescimento tumoral ${ }^{2}$. Como normalmente a doença é diagnosticada em fases tardias, o prognóstico para mãe é ruim, sem relato de sobrevida maior que cinco anos. Enquanto que, para paciente não-grávida, com tumor Dukes C, a sobrevida em cinco anos varia de 30 a $40 \% 5$. Já para o feto, o prognóstico é bom, sendo que não há relato de metástases, e a sobrevida fetal atinge $80 \%{ }^{1}$.

ABSTRACT: Colorectal cancer in pregnancy is a rare condition, associated with a poor prognosis for the woman. The case described is of a 31-year-old female, with an adenocarcinoma of the rectum diagnosed at the $24^{\text {th }}$ week of pregnancy. We present a discuss management in view of updated knowledge and therapeutic approaches.

Keywords: colon cancer; rectal cancer, pregnancy.

\section{REFERÊNCIAS}

1. Cappell MS. Colon cancer during pregnancy. Gastroenterol Clin North Am. 2003;32(1):341-83.

2. Skilling JS. Colorectal cancer complicating pregnancy. Obstet Gynecol Clin North Am. 1998;25(2):417-21.

3. Puig-La Calle Jr, Ng J, Syn GL, Vuolo MA, Guillem JG. Colorectal cancer recurrence during pregnancy - Unique and poorly understood clinical entity. Dis Colon Rectum. 1999;42(5):673-5.

4. Walsh C, Fazio VW. Cancer of the colon, rectum, and anus during pregnancy - The surgeon's perspective. Gastroenterol Clin North Am. 1998;27(1):257-67.

5. Heres P, Wiltink J, Cuesta MA, Burger CW, Van Groeningen CJ, Meijer S. Colon carcinoma during pregnancy: A lethal coincidence. Eur J Obstet Gynecol Reprod Biol. 1993;48:149-52.

6. Bernstein MA, Madoff RD, Caushaj PF. Colon and rectal cancer in pregnancy. Dis Colon Rectum. 1993;36(2):172-8.

7. Balloni L, Pugliese P, Ferrari S, Danova M, Porta C. Colon cancer in pregnancy: report of a case and review of the literature. Tumori. 2000;86(1):95-7.

8. Kömürcü S, Özet A, Öztürk B, Arpaci F, Altundag MK, Tezcan Y. Colon cancer during pregnancy - A case report. J
Reprod Med. 2001;46(1):75-8.

9. Ochshorn Y, Kupferminc MJ, Lessing JB, Pausner D, Geva E, Daniel Y. Rectal carcinoma during pregnancy: a reminder and updated treatment protocols. Eur J Obstet Gynecol Reproduct Biol. 2000;91(2):201-2.

10. Vitoratos N, Salamalekis E, Makrakis E, Creatsas G. Sigmoid colon cancer during pregnancy. Eur J Obstet Gynecol Reproduct Biol. 2002; 104(1):70-2.

11. Caforio L, Draisci G, Ciampelli M, Rossi B, Sollazzi L, Caruso A. Rectal cancer in pregnancy: a new management based on blended anesthesia and monitoring of fetal well being. Eur J Obstet Gynecol Reproduct Biol. 2000;88(1):71-4.

12. Sobrado CW, Mester M, Simonsen OS, Justo CR, deAbreu JN, Habr-Gama A. Retrorectal tumors complicating pregnancy. Dis Colon Rectum. 1996;39(10):1176-9.

13. Minter A, Malik R, Ledbetter L, Winokur TS, Hawn MT, Saif MW. Colon cancer in pregnancy. Cancer Control. 2005;12(3):196-202.

Endereço para correspondência: ANTÔNIO HILÁRIO ALVES FREITAS

Rua Paracatu, 838/206 - Barro Preto CEP: 30180-090 - Belo Horizonte (MG), Brasil

E-mail: hilariofreitas@ig.com.br 